\title{
Prediction and physical analysis of unsteady flows around a pitching airfoil with the dynamic mesh approah
}

\author{
Sébastien Bourdet* — Marianna Braza* — Yannick Hoarau** \\ Rajah El Akoury* — Arif Ashraf* — Gilles Harran* \\ Patrick Chassaing* — Henda Djeridi***
}

* IMFT, UMR 5502 CNRS-INPT, allée du Pr. Camille Soula, F-31400 Toulouse

** IMFS, UMR 7507 ULP-CNRS, 2 rue Boussingault, F-67000 Strasbourg

*** IRENav, EA 3634, BP 600F, 29240 Brest Armées

bourdet@imft.fr

ABSTRACT. The fluid structure interaction due to the pitching motion of a NACA0012 aerofoil has been studied numerically at moderate and high Reynolds numbers. The dynamic mesh method has been employed in the code ICARE/IMFT solving the Navier-Stokes equations in compressible flows. At high Reynolds number, the phase-averaged Navier-Stokes equations have been solved, coupled with advanced URANS modelling in the NSMB code. The vortex dynamics and especially the stall are physically captured by the dynamic mesh method and by the URANS/Organised Eddy Simulation approach.

RÉSUMÉ. L'interaction fluide structure due au mouvement de tangage d'un profil d'aile NACA0012 a été étudiée à des nombres de Reynolds modérés et élevés. La méthode des maillages dynamiques a été employée au sein du code ICARE/IMFT pour la résolution des équations de Navier-Stokes de fluide compressible à nombre de Reynolds modéré. Les équations de Navier-Stokes en moyenne de phase fermées par des schémas URANS avancés pour la modélisation de la turbulence ont été résolues au sein du code NSMB pour des nombres de Reynolds élevés. La dynamique tourbillonnaire, et plus spécialement la dynamique du décrochage, a été bien captée par la méthode des maillages dynamiques et par l'approche de macrosimulation URANS/Organised Eddy Simulation.

KEYWORDS: fluid structure interaction, airfoil, pitching.

MOTS-CLÉS : interaction fluide structure, aile, tangage.

DOI:10.3166/REMN.16.451-476@ 2007 Lavoisier, Paris. Tous droits réservés

REMN - 16/2007. Fluid structure interaction, pages 451 to 476 


\section{Introduction}

This work presents a numerical study and physical analysis of the flow around a NACA0012 pitching airfoil at moderate and high Reynolds numbers. The airfoil movement is an analytical rotation around the pitching axis. This kind of flow simulations allow studying the fluid structure interaction in case of rigid lifting-bodies motion and to analyse physically different kinds of complex phenomena arising from the interaction, such as the buffeting (flapping shock waves) and the dynamic stall (sudden lift loss). Dramatic changes in the aerodynamic performance induced by this phenomenon are of significant interest for rotorcraft or highly maneuverable aircraft, for example. During the motion of the lifting structure, the flow detaches and reattaches over large parts of the body surface. In the pitching case motion of high angle of attack, a highly energetic vortex structure is formed near the leading edge during the increasing angle phase. This structure is convected downstream, along the lifting surface and grows due to the adverse pressure gradient mechanism. During the ascending angle phase, the lift coefficient increases. The vortex structure is convected downstream in the wake, followed by the dynamic stall during the descending angle phase. These steps are characterised by a drastic decrease of lift and of the moment coefficients. A comprehensive review can by found in (McCroskey, 1982). Further experimental studies can be found in (Ahmed et al., 1994, McAlister et al., 1978, McCroskey et al., 1976, Carr et al., 1977, Chandrasekhara et al., 1990, Guo et al., 1994). Numerical simulation of the dynamic stall can be found in (Metha, 1977, Choudhuri et al., 1996, Guo et al., 1994, Barakos et al., 1999) at moderate Reynolds number range. Concerning the high Reynolds number range, the fully developed turbulent flow interacts non-linearly with the coherent vortex structures and yield a very complex dynamic physical process. The outcomes of the european research program UNSI, (Unsteady viscous methods in the context of fluid structure interaction, 1998-2000) where the majority of the european aeronautical industries participated, addressed a comprehensive review of the URANS approaches to simulate the dynamic stall (Haase et al., 2002). The major outcome was that the URANS approaches involving first-order turbulence modelling, including also non-linear behaviour laws, are not yet sufficient to accurately predict the dynamic stall phenomenon. The majority of these approaches used the Arbitrary Lagrangian Eulerian (ALE) approach to take into account the mesh movement.

In the present study an alternative is employed to take into account the domain and grid movement, the dynamic mesh approach, considered as more robust for capturing fast and drastic mesh deformation. This approach was studied by (Batina, 1990). In the present study, an analogy with a spring mesh canvas is used to update the grid, according to (Batina, 1990, Farhat et al., 1998). In this paper, the governing equations of the flow are presented, as well as the methods of mesh motion. The performances of the dynamic mesh will be studied for a moderate-Reynolds number pitching motion (Barakos et al., 1999). Concerning the high-Reynolds number dynamic stall, an advanced URANS approach is employed, able to generate and to capture the stong vortex structures formation and detachment in non-linear interaction with the ran- 
dom turbulence background, the Organised Eddy Simulation, OES approach (Braza, 2002, Braza et al., 2006). The performances will be shown comparing to standard URANS concerning the pitching flow around a NACA0012 airfoil at high Reynolds number. The work carried out in the present paper consists of a first step towards the fluid structure interaction (rigid motion). In our studies in progress, the deformation of the structure in the interaction will be taken into account.

\section{Numerical method}

\subsection{Navier-Stokes equations discretisation scheme and numerical configuration}

The complete time-dependent Navier-Stokes equations have been solved in three dimensions under a conservative form, in a general non-orthogonal curvilinear coordinates system. The Roe upwind scheme (Roe, 1981) has been used to discretise the convection and pressure terms because of their hyperbolic character. The MUSCL approach by (van Leer, 1979) has been employed in order to increase the spatial accuracy from the first to second order. This scheme provide good accuracy and stability as studied in detail by (Bouhadji et al., 2003a). This scheme has been used without limiter. A careful grid refinement has been performed to avoid any spurious wiggle oscillation. However, in the higher Reynolds number ranges, the use of limiters is recommendable to ensure monotonicity with the use of reasonable grid sizes. Diffusion terms have been discretised by central differences and the temporal terms using an explicit, third order of precision in time, three-stage Runge-Kutta TVD scheme (Shu et al., 1988).

The computational domain is a C-Type grid, many grid size had been used, $201 \times$ $59,369 \times 89$ and $501 \times 101$ nodes in order to provide grid-independent solutions. A distance of ten chord-lengths separates the leading edge from the outflow boundary and there are seven chordlengths between the airfoil and the outer boundary. The governing equations and the numerical implementation are described in the Annex, Section 8 .

Free-stream conditions have been imposed at the outer boundaries, except for the downstream one, where a first order extrapolation has been used. On the airfoil surface Neumann conditions are used for the temperature (adiabatic wall), density and energy. Pressure has been computed from the momentum equation which was solved numerically with adherent condition for the velocities. Along the wake line, the boundary values of the velocity, pressure and density have been computed by averaging the mentioned variables from adjacent lines above and below the wake line. Detailed numerical tests have been carried out (Bouhadji, 1998) to ensure that the boundary conditions and the computational domain size do not produce any spurious effect. The numerical characteristics of the present solver ICARE for compressible flows and its behaviour in respect of the boundary conditions have been reported in detail by (Bouhadji et al., 2003b, Bouhadji et al., 2003a, Bouhadji et al., 1997, Bouhadji et al., 1998). We have ensured that the outer boundary was positioned far enough from the airfoil to 
match with simulations that used non-reflecting boundary conditions (Blaschak et al., 1988, Jin et al., 1993).

\subsection{Moving domain equations}

The grid is moving to follow the pitching motion of the solid walls. It is therefore necessary to take into account the velocities of the grid nodes. This is the principle of the dynamic mesh adaptation, achieved by taking into account in the NavierStokes equations two referential systems, the Eulerian and mixte coordinates (Bourdet, 2005, Lefrançois, 1998). Convective fluxes are written in relative velocities taking into account the nodes motion. The first step is to solve the new system of equations. The second step is the updating of the ensemble of metric parameters induced by the grid. To achieve this, it is necessary to update the grid along each new aerofoil position. This is performed by employing a spring mesh canvas analogy: the springs of tension-compression (Batina, 1990) as well as the torsion springs, (Farhat et al., 1998) (Figure 1).

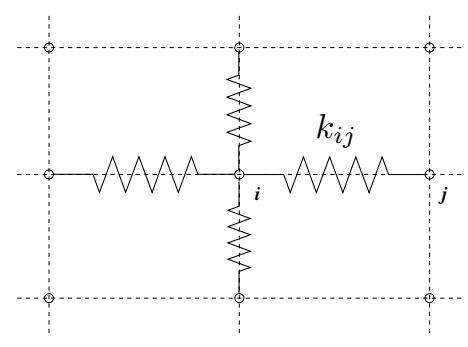

a Tension-compression springs

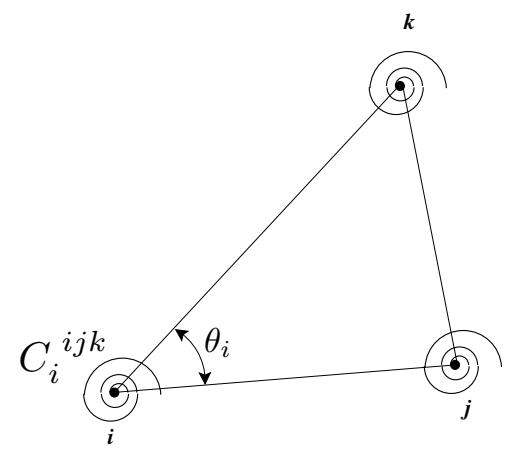

b Torsion springs

Figure 1. Springs analogy for grid updating

The tension-compression springs are fictitious springs that are attached in each segment that links two adjacent grid nodes, (Figure 1a). The spring stiffness, $k_{i j}$, is chosen inversely proportional to the distance between the nodes, $l_{i j}$ of the considered segment:

$$
k_{i j}=\frac{1}{l_{i j}} \quad \text { where } \quad l_{i j}=\phi\left[\left(x_{i}-x_{j}\right)^{2}+\left(y_{i}-y_{j}\right)^{2}\right]^{\alpha},
$$

where $\phi$ and $\alpha$ are adaptative parameters, calibrated numerically to preserve the mesh quality near the wall. Concerning torsion springs, the principle is equivalent: in each edge, $i, j$ et $k$ of a triedron formed by three adjacent grid nodes a torsion spring is associated to a stiffness defined by angular considerations. 
For a node $i$, we define $\theta_{i}$ as the angle between the two segments $i j$ and $i k$ (Figure $1 \mathrm{~b}$ ). On each grid point, a torsion spring is attached for each triangle connected to this point. The torsion spring stiffness is $C_{i}^{i j k}$, defined by:

$$
C_{i}^{i j k}=\frac{1}{\sin ^{2} \theta_{i}}
$$

The first spring type allows avoiding the coincidence between two grid points, because the stiffness increases as the points are approaching each other. The second spring type allows avoiding the passage of grid lines in between the segment.

After each new aerofoil position, the new position of the grid points is defined by solving iteratively the static equilibrium equation of the spring grid canvas. Therefore, the displacement and the velocity of the nodes are determined, as well as the updating of the metrics needed for the Navier-Stokes system solution.

The grid motion can produce in some cases mass sources or sinks. This may contribute to appearance of numerical oscillations. To avoid this, an additional conservation equation has to be solved, the geometrical conservation law (Thomas et al., 1979, Bourdet, 2005). This law is derived from the fact that the temporal variation of

a volume of fluid, whose velocity surface field is $\mathbf{W}_{s}$, has to be equal to the volumes sweeped by the domain oriented surfaces. This law can be written in an analogy to the mass conservation law, as follows:

$$
\frac{d}{d t} \int_{\mathcal{V}} d \mathcal{V}=\int_{S} \mathbf{W}_{s} \cdot d \mathbf{S}
$$

where $\mathcal{V}$ is the volume of the domain considered and $S$ its surface. This equation has been implemented in the whole numerical code ICARE of our team (Bouhadji, 1998). Detailed tests have been carried out to ensure that the results are not affected by numerical perturbations due to the grid movement (Bourdet, 2005).

\section{Flow configuration}

The flow configuration to be examined is the pitching NACA0012 airfoil at $R e_{\infty}=5000$, Mach number $M_{\infty}=0.4$. The pitching motion is imposed around an axis at 0.25 chord length from the leading edge, (Figure 2), according to the oscillation law:

$$
\alpha(t)=\alpha_{0}+\Delta \alpha \sin (\omega t)
$$

where $\alpha_{0}=10^{\circ}$ is the mean angle of attack, $\Delta \alpha=10^{\circ}$ is the oscillation amplitude beyond the mean and $\omega$ the pulsation. The reduced frequency $k=\omega c / 2 U_{\infty}$ of the aerofoil's motion is $k=0.25$. The above test case had been studied numerically by (Barakos et al., 1999), using ALE. 


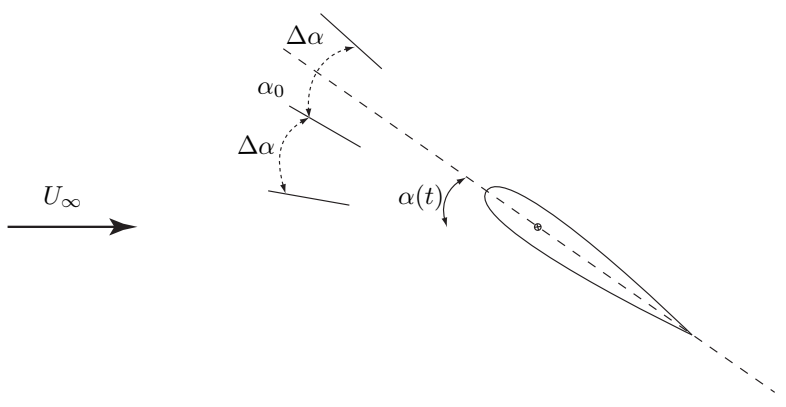

Figure 2. Schematic representation of the pitching airfoil oscillations

\section{Results}

In Figure 3, a comparison of the results by the present study and by the previous one (Barakos et al., 1999), is shown, concerning the lift and moment coefficients. The amplitudes of the lift and moment coefficients simulated by the present study are qualitatively coherent with the results by (Barakos et al., 1999). However, there are significant differences concerning the dynamic stall vortices.
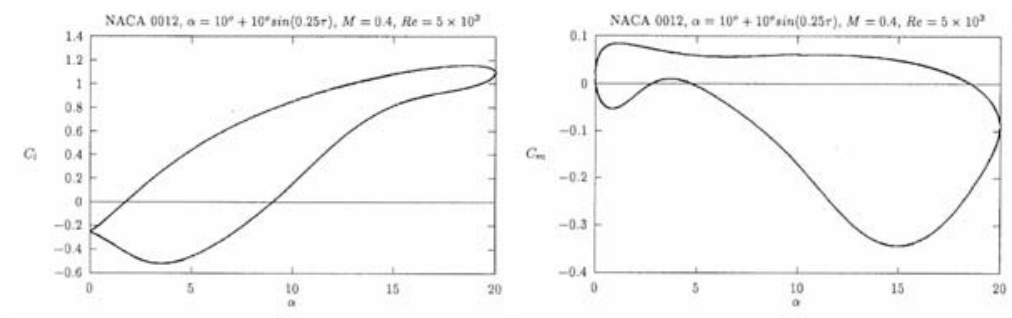

a Results of (Barakos et al., 1999)
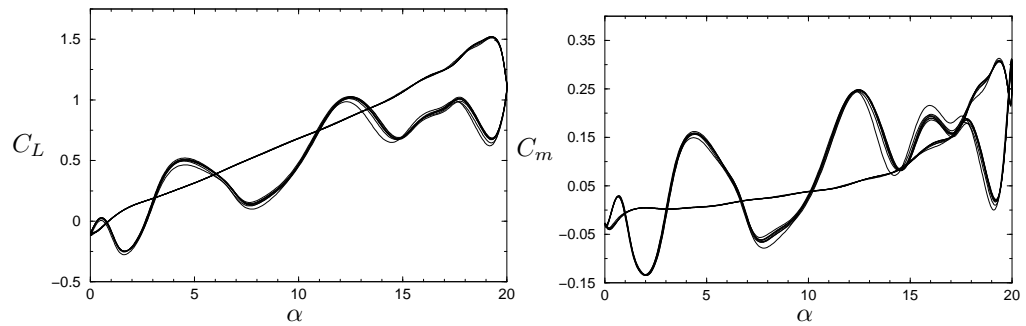

b Results of the present study

Figure 3. Evolution of the lift and moment coefficients, $R e_{\infty}=5000, M_{\infty}=0.4$, $k=0.25$ 
The first difference indicates a drastically different behavior on the kind of dynamic stall. Both studies show a strong hysteresis character during the oscillation cycle, (Figure 3a). The stall occurs at the maximum incidence in the study by (Barakos et al., 1999), whereas it appears at about $19.3^{\circ}$ in the present study, before the maximum angle is reached. The second difference is the formation of a number of secondary peaks in the present study along the suction side, concerning the lift and drag coefficients, (Figure 3b). These peaks correspond to the passage of smaller scale organised eddies along the suction side. Indeed, the vortex dynamics quantified by the present study are more complex than the previous study, where only one vortex structure is convected and creates the dynamic stall.

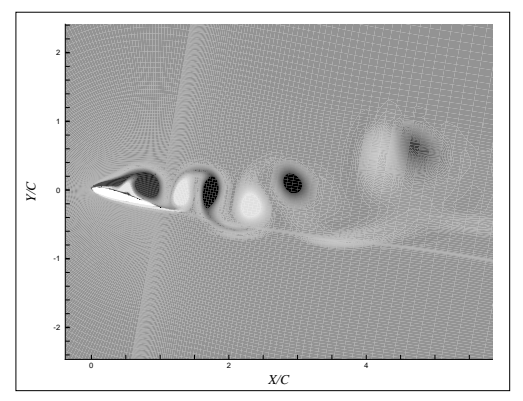

a $t_{\infty}=5.12, \alpha=15.5^{\circ}$

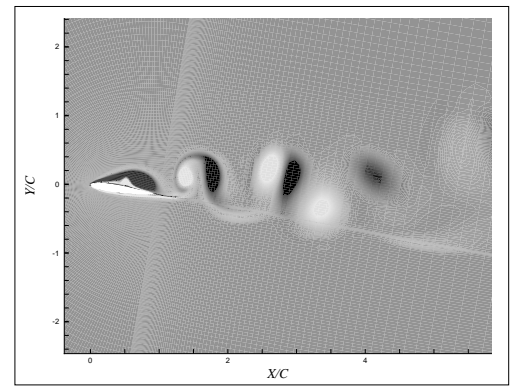

c $t_{\infty}=6.6, \alpha=8.5^{\circ}$

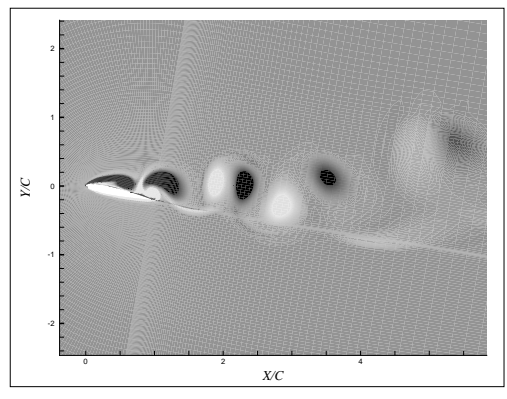

b $t_{\infty}=5.85, \alpha=12.1^{\circ}$

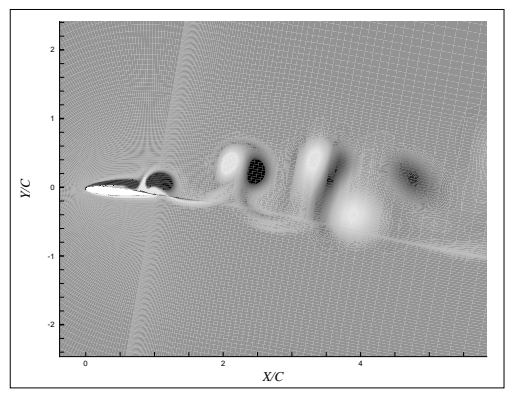

$\mathrm{d} t_{\infty}=7.3, \alpha=5^{\circ}$

Figure 4. Vorticity iso-contours - descendant phase of the pitching motion, $R e_{\infty}=$ $5000, M_{\infty}=0.4, k=0.25$, in white the positive vorticity, in black the negative vorticity

In Figure 4, the vorticity iso-contours are presented at four instantaneous snapshots, allowing tracking of the vortex structures during the flow motion. These structures are not organised along a von-Kármán vortex street, but according to a jet like structure, forming the well known mushroom patterns that characterise the pitching motions (Chandrasekhara et al., 1990), (Choudhuri et al., 1996). The existence of a multitude of vortices beyond the von-Kármán ones in moderate Reynolds number 
range $(R e>2000)$ is also a fact reported by incompressible flow studies around the NACA0012 wing, (Hoarau et al., 2003).

\section{High-Reynolds number pitching motion of the NACA0012 airfoil}

In this section, the high-Reynolds number flow around a NACA0012 airfoil is investigated by using appropriate URANS modelling. This is achieved by means of the Organised Eddy Simulation, OES approach.

\section{The Organised Eddy Simulation approach}

In the context of the URANS/OES macrosimulation (Organised Eddy Simulation (Dervieux et al., 1998, Braza et al., 2006)), the turbulent spectrum is decomposed in a first part regrouping all the coherent processes (resolved part) and in a second part regrouping all the chaotic processes independently on their size (spectrum to be modelled), as presented schematically in Figure 5 and experimentally in Figure 6.

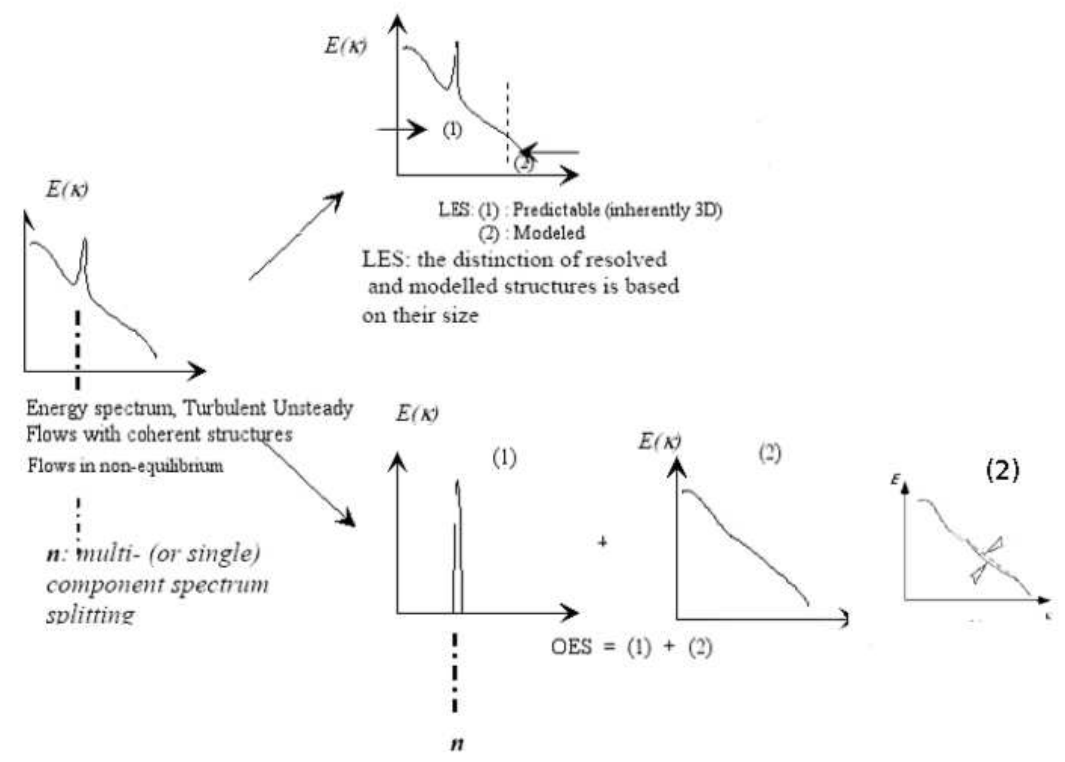

Figure 5. Schematic representation of the energy spectrum in the URANS/Organised Eddy Simulation approach: the distinction between the structures to be resolved and those to be modelled is based upon their organised or random character. Part (2) of the non-equilibrium energy spectrum modelled by reconsidered,advanced statistical turbulence modelling, due to the inertial-range spectrum modulation, schematically shown on the right 


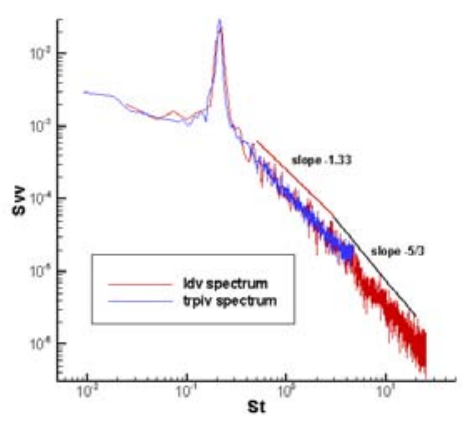

a LDV (Djeridi et al., 2003) and PIV turbulence spectra

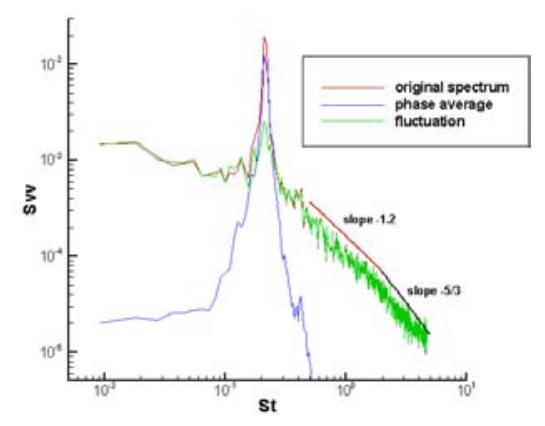

b Time-resolved PIV spectrum

Figure 6. Turbulence spectra in the detached flow past a cylinder at $R_{e}=140000$, $(x / D=1, y / D=0.375)$, (Braza et al., 2006), showing the slope modification in the inertial range due to interaction between the coherent structures and random turbulence

It is recalled that in LES the distinction is done according to the structures size and this limitates this approach to moderate Re-numbers concerning wall-turbulence around bodies. The fact that the spectrum part to be modelled in OES is extended from the low to the high frequencies allows the use of statistical turbulence modelling, that is very efficient in high Reynolds number modelling of wall flows. In the time-domain, the equations are the phase-averaged Navier-Stokes equations, where the turbulent stresses have to be modelled by reconsidered statistical turbulence modelling closures. We had conjectured (Braza, 2002) that due to the non-linear interaction between the coherent part and the incoherent one, there must exist a shape and slope modification of the inertial part in the spectrum, in the vicinity of the peak. This has been now quantified, either by means of the LDV data or by the present study (time-dependent PIV data), (Braza et al., 2006).

The modification of the energy spectrum in the inertial range leads to modified turbulence scales in the context of the statistical two-equation modelling, as achieved in a previous work of us (Jin et al., 1994, Hoarau et al., 2002, Bouhadji et al., 2002), by means of the second-order moment closures. This yields a reconsideration of the eddydiffusion coefficient for the class of two-equation modelling, as well as an improved damping function to attenuate turbulence towards the wall (Jin et al., 1994).

\section{The turbulence modelling}

The Reynolds stress transport model (RSTM) has been used in the phase-averaged Navier-Stokes system, to derive the modification of the turbulence scales involved in 
the evaluation of the eddy-diffusion coefficient $C_{\mu}$ to be used in the OES two-equation modelling. In this study, the computation up to the real wall has been achieved by using the RSTM model (Launder et al., 1975), and (Shima, 1988)'s turbulence damping towards the solid wall. This is an original element of this study in the state of the art, where the RSTM model is difficult to use towards the solid walls because of stability problems, whenever the computational grid is not adequate. Often, zonal approaches are used combining the RSTM in an outer region and a lower-order class of modelling near the wall. However, by using zonal approaches, the ability of the RSTM model of capturing the normal stress anisotropy is lost in the near-region. In the present study, this benefit has been preserved by achieving computation with the RSTM up to the real wall. The second-order turbulence modelling does not use a behavior law for modelling the turbulent stresses. It uses full transport equations for the Reynolds stress tensor, where third-order correlations have to be modelled:

$$
\frac{D \overline{u_{i} u_{j}}}{D t}=D_{i j}^{t}+D_{i j}^{\nu}+P_{i j}+\Pi_{i j}-\varepsilon_{i j}
$$

where the turbulent diffusion is the one proposed by (Daly et al., 1970):

$$
D_{i j}^{t}=\frac{\partial}{\partial x_{k}}\left(C_{s} \frac{k}{\varepsilon} \overline{u_{k} u_{l}} \frac{\partial \overline{u_{i} u_{j}}}{\partial x_{l}}\right)
$$

The viscous diffusion and the production are exact terms:

$$
D_{i j}^{\nu}=\frac{\partial}{\partial x_{k}}\left(\nu \frac{\partial \overline{u_{i} u_{j}}}{\partial x_{k}}\right) \quad P_{i j}=-\left(\overline{u_{i} u_{k}} \frac{\partial \overline{U_{j}}}{\partial x_{k}}+\overline{u_{j} u_{k}} \frac{\partial \overline{U_{i}}}{\partial x_{k}}\right)
$$

The redistribution term is split into the slow part, the rapid part and the wall reflection part as suggested by (Shima, 1988):

$$
\begin{aligned}
\Pi_{i j} & =\Phi_{i j}^{1}+\Phi_{i j}^{2}+\Phi_{i j}^{w} \\
\Phi_{i j}^{1} & =-\gamma\left(P_{i j}-\frac{2}{3} \tilde{P} \delta_{i j}\right) \quad \Phi_{i j}^{2}=-C_{2} \frac{\varepsilon}{k}\left(\overline{u_{i} u_{j}}-\frac{2}{3} k \delta_{i j}\right) \\
\Phi_{i j}^{w} & =f_{w}\left[0.45\left(P_{i j}-\frac{2}{3} \delta_{i j} \tilde{P}\right)-0.03\left(D_{i j}-\frac{2}{3} \delta_{i j} \tilde{P}\right)+0.08 k S_{i j}\right] \\
f_{w} & =\exp \left[-\left(0.015 k^{1 / 2} y / \nu\right)^{4}\right]
\end{aligned}
$$

Finally we have:

$$
\begin{aligned}
& \tilde{P}=\frac{1}{2}\left(P_{i i}\right) \quad \varepsilon_{i j}=\frac{2}{3} \delta_{i j} \varepsilon \\
& D_{i j}=-\left(\overline{u_{i} u_{k}} \frac{\partial U_{k}}{\partial x_{j}}+\overline{u_{j} u_{k}} \frac{\partial U_{k}}{\partial x_{i}}\right)
\end{aligned}
$$


We solve the modified dissipation $\tilde{\varepsilon}=\varepsilon-D$ as proposed by (Chien, 1982):

$\frac{\partial \tilde{\varepsilon}}{\partial t}+\overline{U_{j}} \frac{\partial \tilde{\varepsilon}}{\partial x_{j}}=C_{\varepsilon} \frac{\partial}{\partial x_{i}}\left[\frac{k}{\varepsilon}\left(\overline{u_{i} u_{j}}\right) \frac{\partial \tilde{\varepsilon}}{\partial x_{j}}\right]+\nu \frac{\partial^{2} \tilde{\varepsilon}}{\partial x_{j}^{2}}+\tilde{P} C_{\varepsilon 1} f_{1} \frac{\tilde{\varepsilon}}{k}-C_{\varepsilon 2} f_{2} \frac{\tilde{\varepsilon}^{2}}{k}+D$

with:

$$
D=2 \nu \frac{k}{y^{2}}
$$

The constant used are the classical ones:

$$
C s=0.22 \quad C_{\varepsilon}=0.18 \quad C_{2}=1.8 \quad \gamma=0.6 \quad C_{\varepsilon 1}=1.44 \quad C_{\varepsilon 2}=1.9
$$

A better universality of the employed constants is achieved by the RSTM than with first-order closures. Furthermore, the turbulence production term is provided as a function of the resolved turbulent stresses and therefore it is not submitted to the limitations of the Boussinesq law. A better description of the leading edge region is then achieved, especially with respect to the passage from the laminar to the transitional and afterwards to the turbulent near-wall regions.

The numerical behavior of the RSTM up to the wall in unsteady flow prediction has been carefully studied in the present work. The convergence of the transport equations is achieved using a higher order of iterations within the time step, comparing with the two-equation models. Indeed that the RSTM modelling shows a more unstable character because it is eddy-viscosity free and for this reason it needs numerically a higher number of iterations within a time-step to reach convergence.

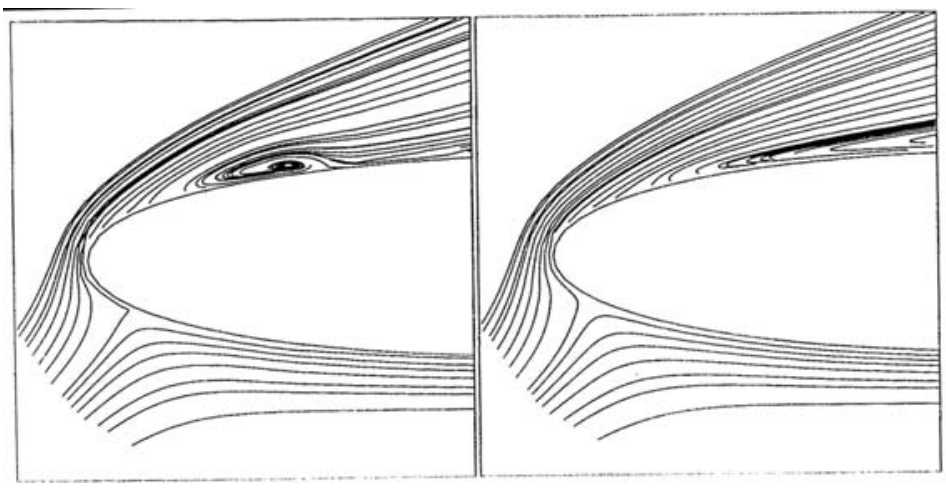

Figure 7. Comparison of the prediction of the leading edge region between two different order closures showing the formation of a laminar steady bubble. Left: Reynolds stress transport model (RSTM) up to the wall (Shima's near-wall version); right: OES modified $k-\varepsilon$ low Reynolds number model of Chien

The flow around a NACA0012 airfoil at $\alpha=20^{\circ}$ and a Reynolds number of $10^{5}$ is computed with the Reynolds Stress model described in the the previous section. A 
very good representation of the physics for the separation bubble (Figure 7) and for the vortex shedding is obtained (Figures 8 and 9).
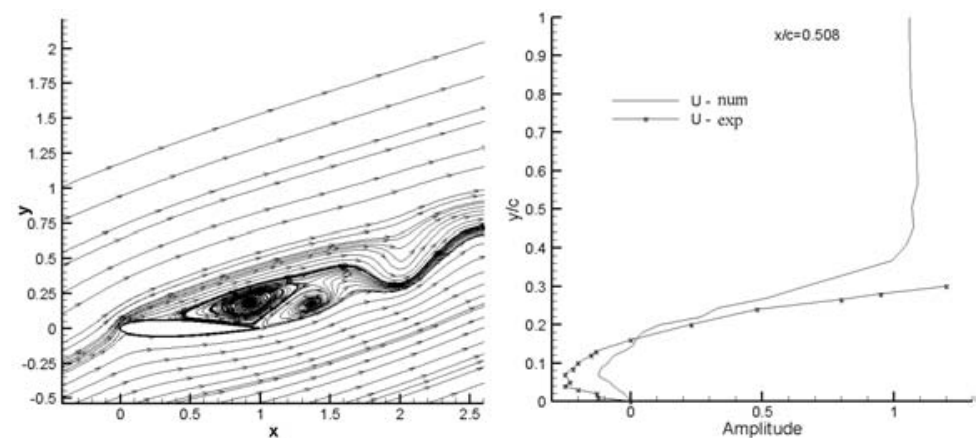

a Instantaneous streamlines at $t^{*}=3 \mathrm{~b}$ Mean velocity profile in the recirculation region

Figure 8. Computation of the flow around a NACA0012 at $R e=10^{5}$ and $\alpha=20^{\circ}$ with the RSTM of (Launder et al., 1975)

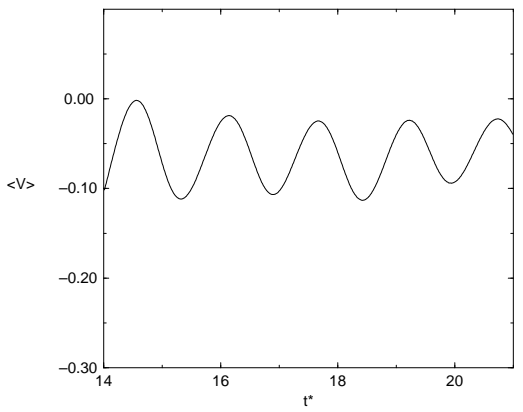

a Time-dependent evolution of $\langle V\rangle$

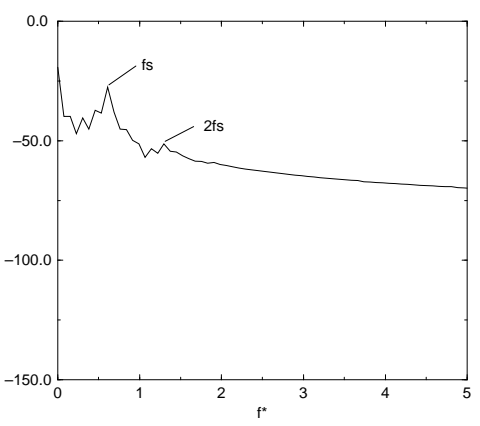

b Spectrum of $\langle V\rangle$

Figure 9. RSTM results for the flow around a NACA0012 airfoil, $R e=10^{5}, \alpha=20^{\circ}$ at the section $x / c=1.226$ and $y / c=0.272$, capturing the inherent unsteadiness and vortex shedding; $\langle V\rangle$ velocity component

\subsection{The eddy-diffusion coefficient}

In the context of two-equation modelling, the unsteady operator of the phaseaveraged Navier-Stokes equations does not suffice for a complete improvement of the prediction of unsteady separated flows if the modelling assumptions are kept the same 
as in fully developed turbulent steady-state flows (in statistical equilibrium). Certainly, the OES approach captures the unsteady organised modes that are expected to develop during the time evolution of the convection term. However, the energy of these modes in the spectrum is broadened (lowered) and in some cases fully damped if the closure hypotheses for the continuous part of the spectrum are not physically adapted. A global effect of this default is seen in the prediction of the mean value and of the amplitude of the fluctuating drag coefficient, both under predicted. This weakness of the two-equation models is strengthened in the case of leading-edge accelerated flows, as those around bodies.

It is well known that these models provide an excessive level of turbulence production (Jin et al., 1994). The excessive rate of kinetic energy in the upstream region of the flow (around the leading edge) comes from the adoption of the Boussinesq approximation: the turbulence stresses are modelled as a linear function of the strain rate by means of the eddy viscosity concept. This modelling comes from a simple analogy and from an extension of the (exact) Boussinesq law that stands for the relation between the molecular stresses and the strain rate under the continuum hypothesis for a Newtonian fluid and therefore derive the well known Navier-Stokes system. The adoption of Boussinesq approximation has given satisfactory results in equilibrium turbulent flows under the (steady) Reynolds-averaged operator. The Boussinesq approximation is therefore satisfactory in the case of a fully accomplished energy cascade from the big to small eddies, where the strain rate and the physical turbulence diffusion are in balance, (e.g. far-field wakes, similarity region of jets, steadystate boundary layers). In the case of leading-edge dominated accelerated flows of the present interest, it is known that the strain-rate is predominant in the vicinity of the leading edge region, where the turbulence diffusion is physically very low. Obviously, the modelling through the Boussinesq law provides a turbulence production, $P_{i j}=\partial\left(<u_{i} u_{j}><U_{i}>\right) / \partial x_{j}$, that is excessively high. This yields an excessively high level of the turbulent kinetic energy, through the modelling relation: $\nu_{t}=C_{\mu} k^{2} / \varepsilon$ where $C_{\mu}=0.09$ after numerical optimisations based on turbulent flows in statistical equilibrium, according to the standard version of the $k-\varepsilon$ model, Figure 10.

The implementation of the RSTM model has offered the possibility to assess an equivalent eddy-diffusivity coefficient to be employed in two-equation models in OES. The cross-term of the turbulence anisotropy tensor has been evaluated by means of the RSTM results (Figure 11). A non-constant behavior of the term $-\langle u v\rangle /\langle k\rangle$ is found in the near-wall region. It will be remembered that this quantity is almost constant in equilibrium boundary layers (Bradshaw et al., 1967) and it has the value of 0.3. Under the equilibrium hypothesis of "Production=Dissipation", the well known $C_{\mu}$ value of 0.09 can be deduced. In the present case of non-equilibrium flow, the variation of $-\langle u v\rangle /\langle k\rangle$ forms a "plateau" at the value of 0.2 (Figure 11). This indicates that the eddy-diffusion coefficient, $C_{\mu}$ should have a lower value than the one of equilibrium boundary layers. 


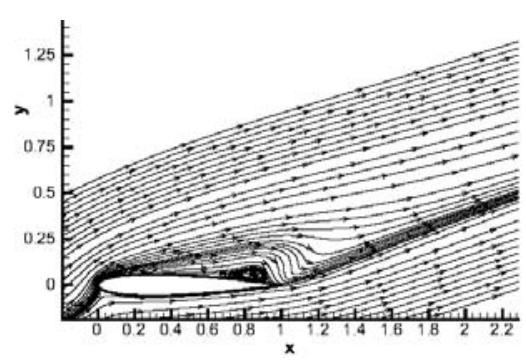

a Streamlines at dimensionless time $t^{*}=$ 18

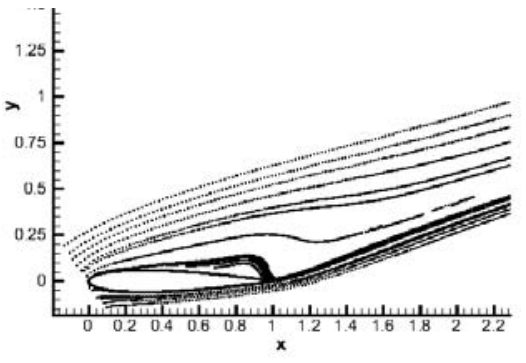

b Streaklines

Figure 10. Computation of the flow around a NACA0012 at $R e=10^{5}$ and $\alpha=20^{\circ}$ with the original version of the $k-\varepsilon$ Chien's model (URANS computation)

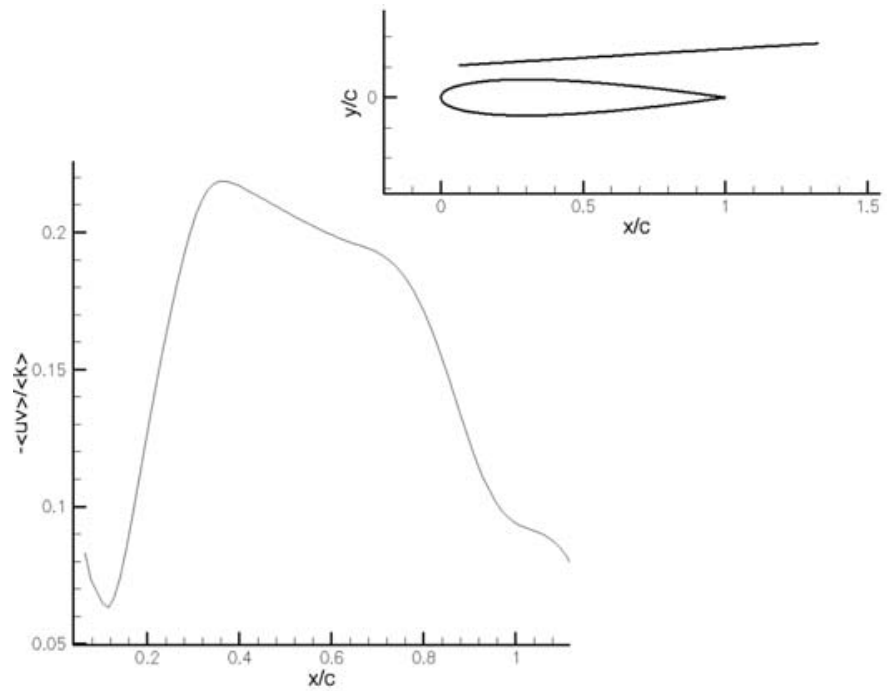

Figure 11. Longitudinal evolution of $-\langle u v\rangle /<k>$ in the recirculation area an in the wake (see sketch)

In the present study, the evaluation of the $C_{\mu}$ coefficient has been possible through an a posteriori quantification of the turbulent kinetic energy and the $\varepsilon$ fields calculated by the RSTM. An equivalent eddy viscosity $\nu_{t}$ has been deduced by adopting a posteriori the Boussinesq law. The optimum value for $C_{\mu}$ has been determined by the relation $\nu_{t}=C_{\mu} k^{2} / \varepsilon$. Table 1 shows the order of magnitude of the $C_{\mu}$ values in the near region. The order of magnitude of $C_{\mu}$ recommendable for massively separated unsteady flows is found 0.02. Experimental results by (Aubrun, 1998) have also indicated this order of magnitude for turbulent flows with coherent structures. 
This value of $C_{\mu}$ as well as the aforementioned modified damping function are used in a modified version of the $k-\varepsilon$ model. Therefore, the model referred as the OES model is the (Chien, 1982) $k-\varepsilon$ model with the two modifications: $C_{\mu}=0.02$ and $f_{\mu}=1-\exp \left(-0.0002 y^{+}-0.000065 y^{+2}\right)$. The behaviour of the modified OES $-k-\epsilon$ model has been tested firstly for a fixed NACA0012 wing at twenty degrees of incidence, developing a massive unsteady separation and vortex shedding, according to the experimental results of (Favier et al., 1998).

Table 1. Evaluation of an equivalent $C_{\mu}$ coefficient by means of the RSTM

\begin{tabular}{|c|c|c|}
\hline $\mathrm{x} / \mathrm{c}$ & $\mathrm{y} / \mathrm{c}$ & equivalent $C_{\mu}$ \\
\hline $3.610^{-2}$ & $3.210^{-2}$ & 0.0158 \\
\hline 0.45 & $5.710^{-2}$ & 0.01859 \\
\hline 0.9064 & 0.15 & 0.01938 \\
\hline 1.41 & -0.678 & 0.0178 \\
\hline 1.23 & 0.11 & 0.0172 \\
\hline 0.73 & 0.19 & 0.024 \\
\hline
\end{tabular}

It is shown that the OES approach is able to predict the separation and detachment, in agreement with the experiment, as well as the frequency of the inherent unsteadiness (experimental Strouhal number 0.51), Figure 9. The mean drag and lift coefficients are shown in Table 2. A good agreement is obtained. Furthermore there is no tendency of over-predicting the drag coefficient, as often occurs with the standard two-equation modelling.

Table 2. Prediction of the aerodynamic coefficients compared to experiments for the flow around a NACA0012 at $R e=10^{5}$ and $\alpha=20^{\circ}$

\begin{tabular}{|c|c|c|}
\hline & Drag & Lift \\
\hline Experience & 0.32006 & 0.75381 \\
\hline OES $k-\varepsilon$ & 0.29355 & 0.86575 \\
\hline Original $k-\varepsilon$ & 0.34248 & 1.04824 \\
\hline
\end{tabular}

Concerning the coherence of this model in respect of the flows in equilibrium, it is worthwhile to notice that the equilibrium behaviour is inherently restituted in the regions where the flow physics reach this state: the flow evolution under the modified modelling is unsteady. The part of the resolved turbulence plus the part of modelled turbulence yield the sum obeying to the equilibrium assumption with an equivalent value of the $C_{\mu}$ coefficient 0.09 . Therefore, the velocity profiles and turbulent stresses calculated after post-treatment of the time-dependent evolution are in accordance with the equilibrium stage in the respective flow regions. 


\subsection{Results}

Computations are performed with the NSMB code (Navier-Stokes multiblock) for the pitching airfoil test-case of (Carr et al., 1977, McCroskey et al., 1976) and (McAlister et al., 1978) at Reynolds number of order one million. The physical parameters of the simulation are given in Table 3. An O-type mesh has been used. The modified OES $k-\epsilon$, and OES $-k-\omega$ turbulence models (Hoarau et al., 2002) are compared with the behaviour of the $k-\omega /$ SST model (Menter, 1994). The computational grid is $500 \times 226$. The unsteady global parameters versus time are shown in Figure 12 and in Figure 13. A good comparison with the experimental data by Mc Croskey and Mc Alistair is achieved. Table 4 shows the maxima and minima of the averaged aerodynamic coefficients in comparison with the experiment. The iso-vorticity contours are shown in Figures 14 and Figure 15 according to the OES $-k-\epsilon$ and $k-\omega /$ SST respectively. The OES $-k-\epsilon$ allows a more rich creation of organised vortices that are inhibited by the $k-\omega / \mathrm{SST}$, especially in the descending phase of the motion. The OES $-k-\omega$ provides practically comparable results with the OES $-k-\epsilon$ model in respect of the iso-vorticity contour dynamics and for the sake of saving space, these results are not presented. The overall comparison between the OES approach and previous URANS one (the SST in this case) indicates the improvements achieved by OES using the same grid size.

Table 3. Physical flow parameters

\begin{tabular}{|l|c|}
\hline Reynolds number & $0.98 \times 10^{6}$ \\
\hline Mean incidence & $15^{\circ}$ \\
\hline Oscillation amplitude $(\Delta \alpha)$ & $10^{\circ}$ \\
\hline Pitch axis location & $1 / 4$ of chord length \\
\hline Oscillation frequency $(\omega)$ & $0.0170383 \mathrm{rad} / \mathrm{sec}$ \\
\hline Reduced drequency & 0.1 \\
\hline Mach number & 0.072 \\
\hline
\end{tabular}

Table 4. Averaged maximum values of the aerodynamic coefficients

\begin{tabular}{|l|c|c|c|c|}
\hline & Experiment & OES $k-\omega$ & OES $k-\varepsilon$ & SST $k-\omega$ \\
\hline$C_{D}(\max )$ & 0.92 & 0.9 & 0.9 & 0.9 \\
\hline$C_{L}(\max )$ & 2.2 & 2.1 & 2.1 & 2.0 \\
\hline$C_{m}(\max )$ & -0.4 & -0.36 & -0.36 & -0.3 \\
\hline pulsation $(\omega)$ & $0.0170383 \mathrm{rad} / \mathrm{sec}$ & & & \\
\hline
\end{tabular}

The fluid structure interaction according to the present study captured by the OES approach yields the formation of a series of smaller scale organised vortices downstream of the leading edge during the ascending phases of the motion, (angles $14.1^{\circ}$ and $17.2^{\circ}$, Figure 14 ). These vortices considerably grow and detach practically from 
the leading edge during the descending phase of the motion. The convected vortex downstream of the trailing edge forms a mushroom like structure, (angle $24.2^{\circ}$ ).
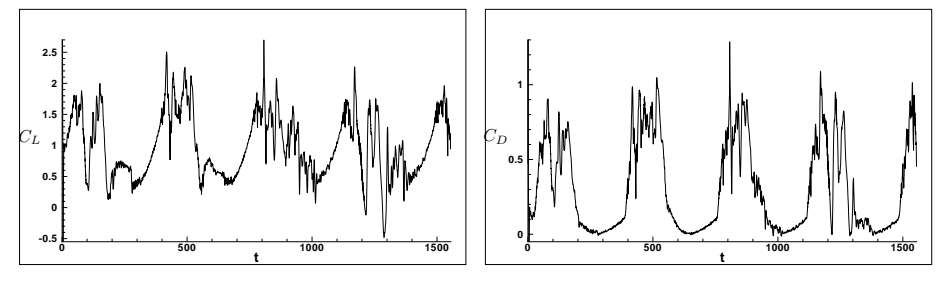

a $C_{\text {Lift }}$

b $C_{D r a g}$

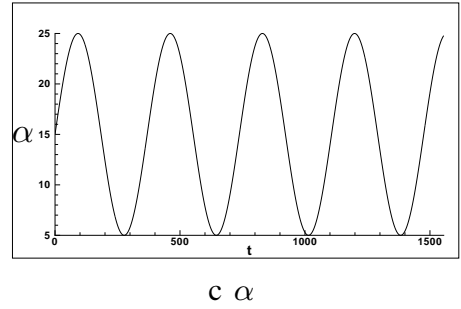

Figure 12. Time-dependent aerodynamic coefficients (drag, lift), $k-\epsilon-O E S$
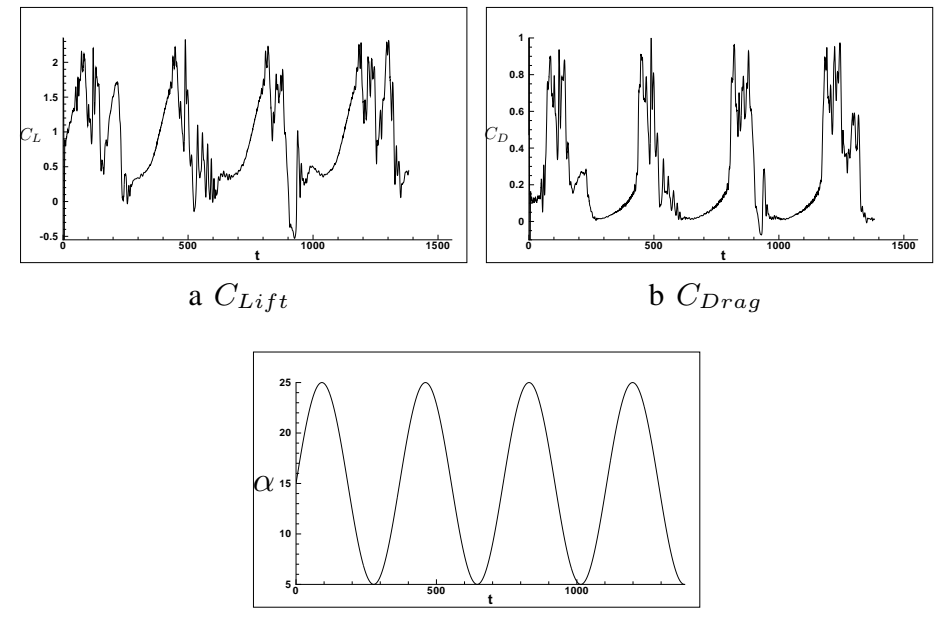

c $\alpha$

Figure 13. Time-dependent aerodynamic coefficients (drag, lift), $k-\omega-S S T$ 


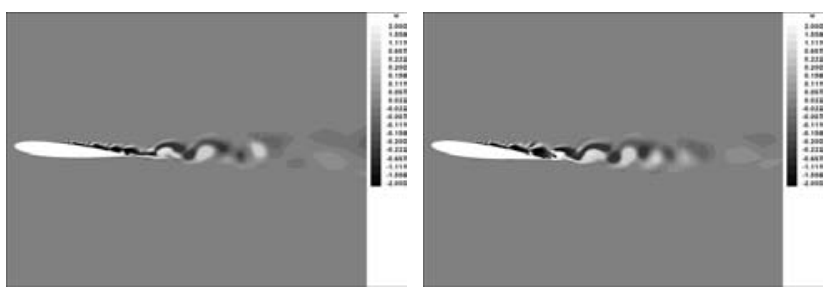

a $6.14^{\circ}$

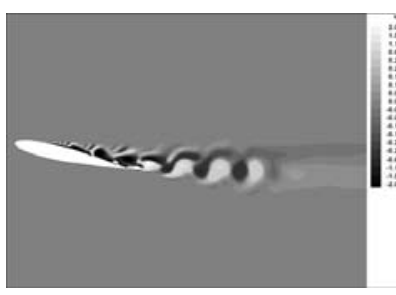

c $14.43^{\circ}$

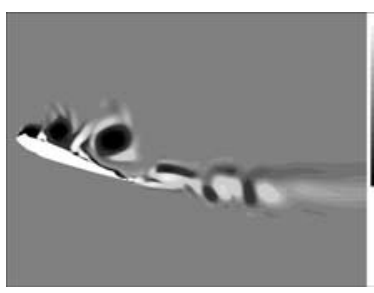

e $22.57^{\circ}$

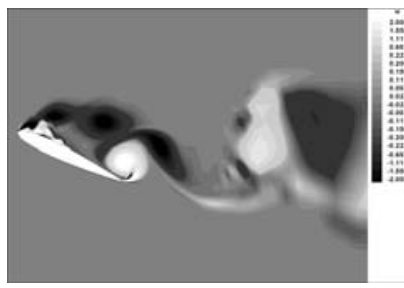

g $24.72^{\circ}$ pitch down b $8.43^{\circ}$

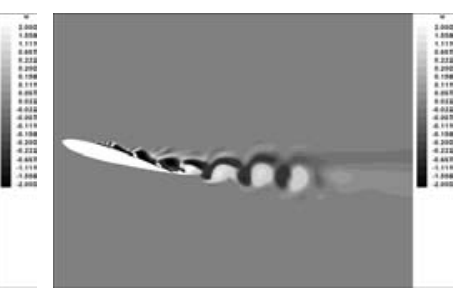

d $17.27^{\circ}$

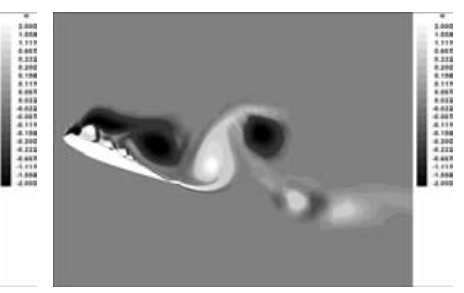

f $24.23^{\circ}$

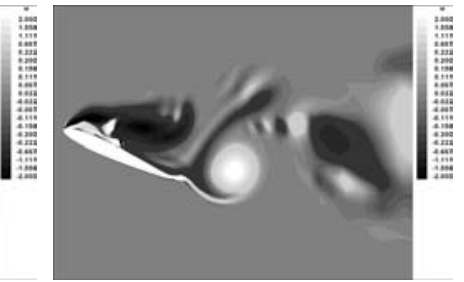

h $23.52^{\circ}$ pitch down

Figure 14. Iso-vorticity contours at different phase angles, $k-\epsilon-O E S$ 

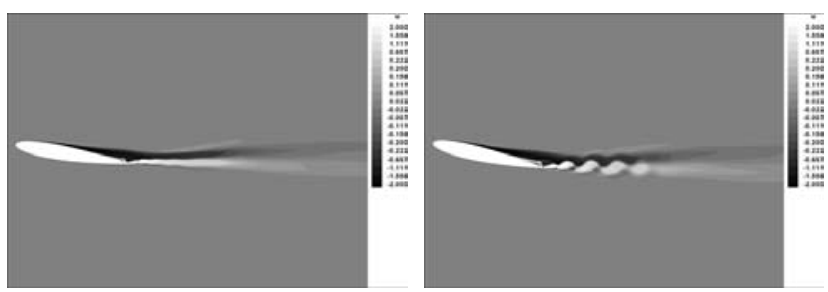

a $10.8^{\circ}$

b $13.8^{\circ}$
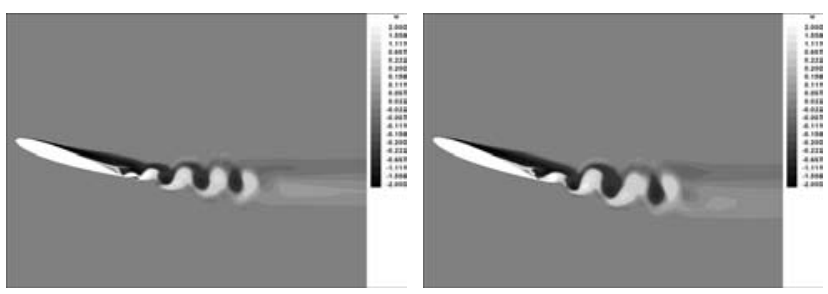

c $19.9^{\circ}$

d $22.3^{\circ}$
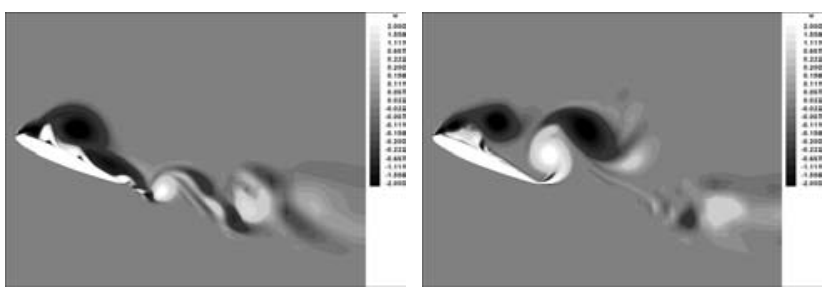

e $24.9^{\circ}$

f $24.8^{\circ}$ pitch down
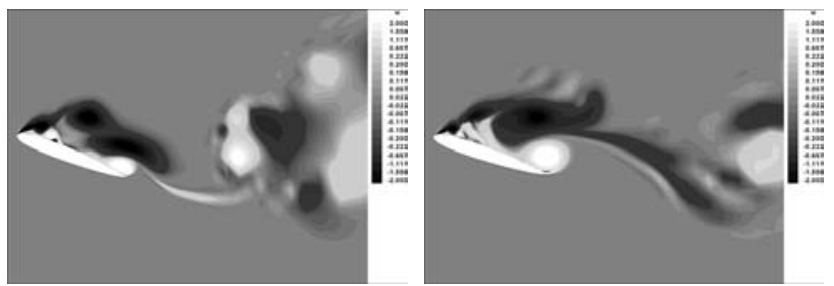

g $21.7^{\circ}$ pitch down

h $19.1^{\circ}$ pitch down

Figure 15. Iso-vorticity contours at different phase angles, $k-\omega-S S T$ 


\section{Conclusion}

The dynamic mesh adaptation method indicates a robust behavior and provides results that capture the fluid structure interaction dynamics in the case of pitching airfoils motion, providing rich vortex dynamics in accordance with experimental visualisations. The numerical approach by this method indicates achievement of more accurate dynamic stall phenomenon in respect to the physics. The OES macrosimulation approach provides a good representation of the vortex dynamics concerning the interaction at high Reynolds number turbulent pitching flow around the NACA0012 airfoil, with extrema aerodynamic parameters close to the experiment. This approach provides a more rich coherent structures dynamics in the formation and the stall phases than previous URANS approaches.

\section{Acknowledgements}

The authors are grateful to Dr. Jan Vos (Computational Fluids and Structures Engineering (CFS)-Lausanne) for his technical assistance concerning the NSMB consortium code in which the IMFT's research Team of Fluid Structure Interaction participates. Part of the CPU time allocation has been supplied by the french goverment supercomputing centres CINES, IDRIS and CALMIP. Part of this work is supported by the European Community Research Programme in Aeronautics, 'DESIDER', Detached Eddy Simulation for Industrial Aerodynamics, $\mathrm{N}^{\circ}$ AST3-CT-2003-502842, coordinated by EADS. The authors are grateful to Dr. Werner Haase (EADS, coordinator of DESIDER) and to Dr. Alessandro D'Alassio (EUROCOPTER, DESIDER partner) for their valuable discussions concerning the dynamic stall.

\section{References}

Ahmed S., Chandrasekhara M., « Reattachment studies of an oscillating airfoil dynamic stall flowfield », AIAA J., vol. 32, n 5, p. 1006-1012, 1994.

Aubrun S., «Etude expérimentale des structures cohérentes dans un écoulement turbulent décollé et comparaison avec une couche de mélange », Thèse de Doctorat, Institut National Polytechnique de Toulouse, France, 1998.

Barakos J., Drikakis G. A., « An implicit unfactored method for unsteady turbulent compressible flow with moving boundaries », Comp. Fluids, vol. 28, p. 899-922, 1999.

Batina J., « Unsteady Euler airfoil solutions using unstructured dynamic meshes », AIAA J., vol. 28, n 8, p. 1381-1388, august, 1990.

Blaschak J., Kriegsmann G. A., « A comparative study of absorbing boundary conditions », J. Comp. Phys., vol. 77, p. 79-130, 1988.

Bouhadji A., Analyse physique par simulation numérique de phénomènes de transition Bi et Tridimensionnels dans l'écoulement compressible, visqueux autour d'une aile d'avion, Thèse de doctorat, Institut National Polytechnique de Toulouse, 1998. 
Bouhadji A., Bourdet S., Braza M., Hoarau Y., Rodes P., G.Tzabiras, « Turbulence Modelling of Unsteady Flows with a Pronounced Periodic Character », Notes on Numerical Fluid Mechanics, vol. 81, p. 87-96, 2002.

Bouhadji A., Braza M., «Compressibility effect on the 2D and 3D vortex structures in a transonic flow around a wing », ERCOFTAC bulletin, vol. 34, p. 4-9, 1997.

Bouhadji A., Braza M., «Physical analysis of unsteady viscous flow phenomena around a wing by Direct Navier-Stokes Simulation », 4th ECCOMAS Computational Fluid Dynamics Conference, Athens, 1998.

Bouhadji A., Braza M., « Organised modes and shock-vortex interaction in unsteady viscous transonic flows around an aerofoil. Part II:Reynolds number effect », J. Computers and Fluids, vol. 32, n 9, p. 1261-1281, $2003 \mathrm{a}$.

Bouhadji A., Braza M., « Physical analysis by numerical simulation of organised modes and shock-vortex interaction in transonic flows around an aerofoil. Part I: Mach number effect », J. Computers and Fluids, vol. 32, n 9, p. 1233-1260, 2003b.

Bourdet S., Analyse physique d'écoulements compressibles instationnaires autour de structures portantes dans le contexte d'interaction fluide-structure, Thèse de doctorat, Institut National Polytechnique de Toulouse, 2005.

Bradshaw P., Atwell N., Ferris D., «Calculation of boundary layer development using the turbulent energy equation », J. of Fluid Mech., vol. 28, p. 593-616, 1967.

Braza M., «Flow Physics Modelling in Fluid-Structure Interaction », Notes on Numerical Fluid Mechanics, dedicated volume "Progress in Computational Flow-Structure Interaction ", Sci. Eds. W. Haase, V. Selmin, B. Winzell, Publisher Springer, vol. 81, p. 145-154, 2002.

Braza M., Perrin R., Hoarau Y., « Turbulence Properties in the cylinder wake at high Reynolds number », J. Fluids. Struct., vol. in print, p. 1-16, 2006.

Carr L., McAlister K., McCroskey W., Analysis of the developpment of dynamic stall base on oscillating airfoils experiments, Technical Notes $n^{\circ}$ TN-D-8382, NASA, 1977.

Chandrasekhara M., Carr L., «Flow visualisations studies of the Mach number effets on dynamic stall », J. Aircraft, vol. 27, p. 516-522, 1990.

Chien K., «Predictions of channel and boundary-layer flows with a low-Reynolds number turbulence model », AIAA Journal, vol. 20, p. 33-38, 1982.

Choudhuri G., Knight D., « Effects of compressibility, pitch rate, and Reynolds number on unsteady incipient leading -edge boundary layer separation over a pitching airfoil », J. Fluid Mech., vol. 308, p. 195-217, 1996.

Daly B., Harlow F., « Transport equations in turbulence », Physics of Fluid, vol. 13, p. 26342649, 1970.

Dervieux A., Braza M., Dussauge J., « Computation and comparison of efficient turbulence models in aeronautics », Notes on Numerical Fluid Mechanics, vol. 65, Vieweg, 1998.

Djeridi H., Braza M., Perrin R., Harran G., Cid E., Cazin S., « Near-Wake Turbulence Properties Around a Circular Cylinder at High Reynolds Number », J. Flow Turbulence and Combustion, vol. 71, p. 19-34, 2003.

Farhat C., Degand C., Koobus B., Lesoinne M., « Torsional springs for two-dimensional dynamics unstructured fluid meshes », Comput. Methods Appl. Mech. Engrg., vol. 163, p. 231-245, 1998. 
Favier D., Maresca C., Berton E., Allain C., « Etude Expérimentale et Numérique Du Développement de la Couche Limite Instationnaire sur Modèles Oscillants En Écoulements 2D/3D », D.R.E.T., Convention no 95-052, Rapport final de synthèse, Mai, 1998.

Guo W., Fu D., Ma Y., « Numerical investigation of dynamic stall of an oscillating airfoil », Int. J. Numer. Methods Fluids, vol. 10, p. 723-734, 1994.

Haase W., Selmin V., Winzell B., « Progress in Computational Flow-Structure Interaction, Results of the Project UNSI, Supported by the European Union 1998 - 2000 », Notes on Num. Fluid Mech. and Multidisciplinary Design, 2002.

Hoarau Y., Braza M., Ventikos Y., Faghani D., Tzabiras G., « Organized modes and the three-dimensional transition to turbulence in the incompressible flow around a NACA0012 wing », J. Fluid Mech., vol. 496, p. 63-72, 2003.

Hoarau Y., Favier D., Braza M., P.Rodes, G.Tzabiras, Allain C., Berton E., Maresca C., « Turbulence Modelling of Unsteady Flows with a Pronounced Periodic Character », IUTAM Symposium on Unsteady Separated Flows, 8-12 April 2002, Toulouse, France, 2002.

Jin G., Braza M., « A non-reflecting outlet boundary condition for incompressible unsteady Navier-Stokes calculations », J. Comp. Phys., vol. 107, p. 239-253, 1993.

Jin G., Braza M., « A Two-equation turbulence model for unsteady separated flows around airfoils », AIAA Journal, vol. 32, p. 2316-2320, 1994.

Launder B., Reece G., Rodi W., « Progress in the development of a Reynolds-stress turbulence closure », J. of Fluid Mech., vol. 68, p. 537-566, 1975.

Lefrançois E., Modèle numérique de couplage fluide-structure pour l'étude des phénomène aéroélastiques avec applications aux moteurs fusée, Thèse de doctorat, Université de Rouen, 1998.

McAlister K., Carr L., McCroskey W., Dynamic stall experiments on the NACA0012 airfoil, Technical Paper $n^{\circ}$ TP 1100, NASA, 1978.

McCroskey W., « Unsteady airfoils », Ann. Rev. Fluid. Mech., vol. 14, p. 285-311, 1982.

McCroskey W., Carr L., McAlister K., « Dynamic stall experiments on oscillating airfoils », AIAA J., vol. 14, p. 57-63, 1976.

Menter F., « Two-Equation Eddy-Viscosity Turbulence Models for Engineering Applications », AIAA Journal, vol. 32, n 8, p. 1598-1605, 1994.

Metha U., Dynamic stall of an oscillating airfoil, Report n CP-227, AGARD, 1977.

Roe P., « Approximate Riemann solvers, parameter vectors and difference schemes », J. Comp. Phys., vol. 43, p. 357-372, 1981.

Shima N., « A Reynolds-stress model for near-wall and low-Reynolds-number regions », J. of Fluids Engng, vol. 110, p. 38-44, 1988.

Shu C., Osher S., « Efficient implementation of essentially non-oscillatory shock-capturing schemes », J. Comp. Phys., vol. 77, p. 439-471, 1988.

Thomas P., Lombard C., « Geometric conservation law and its application to flow computations on moving grids », AIAA J., vol. 17, n 10, p. 1030-1037, 1979.

van Leer B., «Towards the ultimate conservative difference scheme: a second-order sequel to Godunov's method », J. Comp. Phys., vol. 32, p. 101-136, 1979.

Yee H., Upwind and symmetric shock-capturing schemes, Technical Memorandum $\mathrm{n}^{\circ} \mathrm{TM}-$ 89464, NASA, May, 1987. 


\section{Annex: governing equations and numerical implementation}

\subsection{Governing equations}

In two dimensions, the Navier-Stokes equations for an unsteady compressible flow can be written under conservative and non-dimensional form, in a general, nonorthogonal, curvilinear coordinate system:

$$
\begin{aligned}
& \frac{\partial \widehat{q}}{\partial t}+\frac{\partial \widehat{E}}{\partial \xi}+\frac{\partial \widehat{F}}{\partial \eta}=\frac{1}{R e}\left(\frac{\partial \widehat{G}}{\partial \xi}+\frac{\partial \widehat{R}}{\partial \eta}\right) \\
& \widehat{q}=J\left[\begin{array}{c}
\rho \\
\rho u \\
\rho v \\
e
\end{array}\right] \widehat{E}=J\left[\begin{array}{c}
\rho u U+\xi_{x} p \\
\rho v U+\xi_{y} p \\
(e+p) U
\end{array}\right] \widehat{F}=J\left[\begin{array}{c}
\rho V \\
\rho u V+\eta_{x} p \\
\rho v V+\eta_{y} p \\
(e+p) V
\end{array}\right] \\
& \widehat{G}=J\left[\begin{array}{c}
0 \\
\xi_{x} \tau_{x x}+\xi_{y} \tau_{x y} \\
\xi_{x} \tau_{y x}+\xi_{y} \tau_{y y} \\
\xi_{x} \beta_{x}+\xi_{y} \beta_{y}
\end{array}\right] \widehat{R}=J\left[\begin{array}{c}
0 \\
\eta_{x} \tau_{x x}+\eta_{y} \tau_{x y} \\
\eta_{x} \tau_{y x}+\eta_{y} \tau_{y y} \\
\eta_{x} \beta_{x}+\eta_{y} \beta_{y}
\end{array}\right]
\end{aligned}
$$

where $\widehat{q}$ is the unknown vector, $\widehat{E}, \widehat{F}$ and $\widehat{G}, \widehat{R}$ are the inviscid and the viscous fluxes, respectively. $u, v$ are the cartesian velocity components in $\mathrm{x}$ and $\mathrm{y}$ direction, $\rho$ the density, $p$ the pressure and $e$ the total energy per unit volume, defined as:

$$
e=\rho e_{i}+\rho \frac{u^{2}+v^{2}}{2}
$$

where $e_{i}$ is the specific internal energy.

$U$ and $V$ are the so-called contravariant velocities along $\xi$ and $\eta$ :

$$
U=\xi_{x} u+\xi_{y} v \quad V=\eta_{x} u+\eta_{y} v
$$

The stresses terms are given as:

$$
\begin{aligned}
& \tau_{x x}=\frac{\mu}{3}\left(4 u_{x}-2 v_{y}\right) \quad \tau_{y y}=\frac{\mu}{3}\left(4 v_{y}-2 u_{x}\right) \\
& \tau_{x y}=\tau_{y x}=\mu\left(u_{y}+v_{x}\right)
\end{aligned}
$$


where:

$$
u_{x}=u_{\xi} \xi_{x}+u_{\eta} \eta_{x} \quad u_{y}=u_{\xi} \xi_{y}+u_{\eta} \eta_{y}
$$

we define $\beta_{x}$ and $\beta_{y}$ as:

$$
\beta_{x}=\frac{\gamma \mu}{P_{r}} e_{i_{x}}+u \tau_{x x}+v \tau_{x y} \quad \beta_{y}=\frac{\gamma \mu}{P_{r}} e_{i_{y}}+u \tau_{y x}+v \tau_{y y}
$$

$R_{e}, P_{r}, \gamma$ and $\mu$ are respectively, the Reynolds number, the Prandtl number, the ratio of specific heat, and the dynamic viscosity. We can define the dimensionless laminar viscosity by the Sutherland law as:

$$
\mu=\frac{\frac{110.4}{T_{0}}+1}{\frac{110.4}{T_{0}}+T} T^{\frac{3}{2}}
$$

Where $T_{0}$ is a reference temperature.

The metric terms are :

$$
\xi_{x}=\frac{y_{\eta}}{J} \quad \eta_{x}=-\frac{y_{\xi}}{J} \quad \xi_{y}=-\frac{x_{\eta}}{J} \quad \eta_{y}=\frac{x_{\xi}}{J}
$$

where $J=x_{\xi} y_{\eta}-x_{\eta} y_{\xi}$ is the jacobian of the transformation. Finally, the pressure $p$ is related to $e_{i}$ and $\rho$ by the perfect gas equation of state:

$$
p=(\gamma-1) \rho e_{i}
$$

The dimensionless variables are specified through the chord length, the uniform stream velocity, and density.

\subsection{Numerical method}

\subsubsection{The spatial scheme}

The convection-pressure terms, because of their hyperbolic character, are responsible of weak solutions; their discretisation should be sufficiently stable to capture flow discontinuities (shock waves) without diminishing the accuracy of the scheme. The Roe's upwind scheme (Roe, 1981) among other, is appropriately adapted to the capture of shock waves and has moreover good properties of stability. 
The discretisation of the $\xi$-directional inviscid term $\widehat{E}$ gives:

$$
\left(\frac{\partial \widehat{E}}{\partial \xi}\right)_{i j}=\frac{\widehat{E}_{i+\frac{1}{2} j}^{*}-\widehat{E}_{i-\frac{1}{2} j}^{*}}{\Delta \xi}
$$

where $\widehat{E}^{*}$ is the numerical flux, and it is expressed as:

$$
\widehat{E}_{i+\frac{1}{2} j}^{*}=\frac{1}{2}\left[\widehat{E}_{i+\frac{1}{2} j}^{r}+\widehat{E}_{i+\frac{1}{2} j}^{l}-\mathcal{D}_{i+\frac{1}{2} j}\right]
$$

The damping function $\mathcal{D}$ has the following form:

$$
\mathcal{D}_{i+\frac{1}{2} j}=|\overline{\mathcal{A}}|_{i+\frac{1}{2} j}\left[\widehat{q}_{i+\frac{1}{2} j}^{r}-\widehat{q}_{i+\frac{1}{2} j}^{l}\right]
$$

where $\overline{\mathcal{A}}$ is the Roe's matrix.

Following the MUSCL ${ }^{1}$ approach (van Leer, 1979), one can obtain a spatially higherorder differencing by taking $q^{l}$ and $q^{r}$ as follow:

$$
\begin{aligned}
& q_{i+\frac{1}{2} j}^{l}=q_{i j}+\frac{1}{2}\left[(1-\beta) \Delta q_{i+\frac{1}{2} j}+\beta \Delta q_{i-\frac{1}{2} j}\right] \\
& q_{i+\frac{1}{2} j}^{r}=q_{i+1 j}-\frac{1}{2}\left[(1-\beta) \Delta q_{i+\frac{1}{2} j}+\beta \Delta q_{i+\frac{3}{2} j}\right]
\end{aligned}
$$

where $\Delta q_{i+\frac{1}{2} j}=q_{i+1 j}-q_{i j}, q=J^{-1} \widehat{q}$, and $\beta \in[0,1]$.

By taking $\beta=\frac{1}{2}$, a second-order accurate half-upwind scheme or the so called Fromm scheme is adopted in this study (see (Yee, 1987)).

\subsubsection{The temporal scheme}

The explicit third-stage third-order Runge-Kutta scheme (Shu et al., 1988) is used. Re-writting th Navier-Stokes equations as:

$$
\frac{\partial \widehat{q}}{\partial t}=L(\widehat{q})
$$

1. Monotonic Upstream Schemes for Conservation Laws. 
this scheme reads:

$$
\begin{aligned}
& \widehat{q}_{i j}^{(0)}=\widehat{q}_{i j}^{(n)} \\
& \left\{\begin{array}{l}
\widehat{q}_{i j}^{(1)}=\widehat{q}_{i j}^{(0)}+\frac{\Delta t}{4} L\left(\widehat{q}_{i j}^{(0)}\right) \\
\widehat{q}_{i j}^{(2)}=\widehat{q}_{i j}^{(0)}+\frac{\Delta t}{4} L\left(\widehat{q}_{i j}^{(0)}\right)+\frac{\Delta t}{4} L\left(\widehat{q}_{i j}^{(1)}\right) \\
\widehat{q}_{i j}^{(3)}=\widehat{q}_{i j}^{(0)}+\frac{\Delta t}{6} L\left(\widehat{q}_{i j}^{(0)}\right)+\frac{\Delta t}{6} L\left(\widehat{q}_{i j}^{(1)}\right)+\frac{2 \Delta t}{3} L\left(\widehat{q}_{i j}^{(2)}\right) \\
\widehat{q}_{i j}^{(n+1)}=\widehat{q}_{i j}^{(3)}
\end{array}\right.
\end{aligned}
$$

The advantage of Runge-Kutta scheme lies in the fact that the spatial discretisation is totally cut out of the temporal one; this ensure us an appreciable and non-negligible freedom in the choice of the numerical spatial scheme. 